\title{
Butyrate-mediated acquisition of chemoresistance by human colon cancer cells
}

\author{
HYANG RI KANG ${ }^{1}$, HYEON GYEOM CHOI ${ }^{2}$, CHAE KYUNG JEON ${ }^{3}$, SOO-JEONG LIM ${ }^{4}$ and SO HEE KIM ${ }^{1}$ \\ ${ }^{1}$ College of Pharmacy and Research Institute of Pharmaceutical Science and Technology, Ajou University, Suwon; \\ ${ }^{2}$ College of Natural Sciences, Hannam University, Daejeon, Republic of Korea; ${ }^{3}$ Grinnell College, Grinnell, \\ IA 50112, USA; ${ }^{4}$ Department of Bioscience and Biotechnology, Sejong University, Seoul, Republic of Korea
}

Received February 6, 2016; Accepted May 10, 2016

DOI: $10.3892 /$ or.2016.4838

\begin{abstract}
Butyrate is a short-chain fatty acid produced by the intestinal microflora and it not only induces apoptosis but also inhibits the proliferation of cancer cells. Recently, it has been reported that butyrate may cause resistance in colon cancer cells. Therefore, we investigated the effects of increased resistance to butyrate in HCT116 colon cancer cells. We established HCT116 cells resistant to butyrate (HCT116/ BR) by treating HCT116 parental cells (HCT116/PT) with increasing concentrations of butyrate to a maximum of $1.6 \mathrm{mM}$ for 3 months. The butyrate concentrations that inhibited cell growth by $50 \%\left(\mathrm{IC}_{50}\right)$ were 0.508 and $5.50 \mathrm{mM}$ in HCT116/PT and HCT116/BR cells. The values after treatment with paclitaxel, 5-fluorouracil (5-FU), doxorubicin and trichostatin A (TSA) were 2.42, 2.36, 4.31 and 11.3-fold higher, respectively, in HCT116/BR cells compared with HCT116/PT cells. The protein expression of drug efflux pumps, such as P-glycoprotein (P-gp), breast cancer-resistant protein (BCRP) and the multidrug resistance associated protein 1 (MRP1), did not differ between HCT116/PT and HCT116/BR cells. The expression level of the anti-apoptotic Bcl-xL protein was increased while those of pro-apoptotic Bax and Bim proteins were reduced in HCT116/BR cells. There were no significant differences in cell motility and invasion. This study suggests that exposure of colon cancer cells to butyrate results in development of resistance to butyrate, which may play a role in the acquisition of chemoresistance in colon cancer.
\end{abstract}

\section{Introduction}

Colon cancer is one of the most common cancers and its prevalence is increasing gradually due to changes in lifestyle (1).

Correspondence to: Dr So Hee Kim, College of Pharmacy and Research Institute of Pharmaceutical Science and Technology, Ajou University, Suwon 443-749, Republic of Korea

E-mail:shkim67@ajou.ac.kr

Key words: colon cancer, butyrate, chemoresistance, apoptosis, HCT116 cells
Epidemiological studies have attempted to elucidate an association between a high-fiber diet and decreased incidence and progression of colon cancer. Butyrate is a short-chain fatty acid that is naturally produced by the intestinal microflora during dietary fiber fermentation and has been suggested as a target for colon cancer therapy because it inhibits cell proliferation and causes apoptosis in colon cancer cells (2). Butyrate suppresses cell proliferation by causing cell cycle arrest at the $G_{1}$ and $G_{2}$ phases, which involves cyclin D1 and retinoblastoma ( $\mathrm{Rb}$ ) signaling (3). In addition, butyrate is a histone deacetylase (HDAC) inhibitor, similar to trichostatin A(TSA), and results in apoptosis by increasing the expression of pro-apoptotic proteins, such as Bak, Bad and Bim, and decreasing that of anti-apoptotic proteins, such as Bcl-2 and Bcl-xL (4).

Butyrate plays an important role in normal colonocytes as a major energy source and promotes their proliferation (5). However, most cancer cells prefer to utilize glucose as an energy source even under abundant oxygen conditions, so colon cancer cells cannot use butyrate efficiently, which enhances its anticancer effects (6).

Recently, it has been reported that butyrate may trigger resistance in colon cancer cells (7). As mentioned above, butyrate is a natural product of dietary fiber fermentation to which colonocytes are consistently exposed. Acquisition of resistance to butyrate can affect the proliferation, cell cycle and invasiveness of colon cancer cells. However, the mechanism of resistance to butyrate is unclear. Drug efflux pumps, such as P-glycoprotein (P-gp), breast cancer-resistant protein (BCRP) and the multidrug resistance associated protein 1 (MRP1), may be related to the acquisition of resistance to butyrate. Such proteins are considered to influence drug absorption, bioavailability and resistance (8).

In this study, we established a butyrate-resistant colon cancer cell line and investigated the effect of butyrate resistance in colon cancer cells, focusing on sensitivity to chemotherapeutic agents, the cell cycle and invasiveness.

\section{Materials and methods}

Chemicals. Sodiumbutyrate, TSA, mitomycin C,3-(4,5-dimethylthiazol-2-yl)-2,5-diphenyltetrazolium-bromide (MTT) and protease inhibitor cocktail were purchased from Sigma-Aldrich (St. Louis, MO, USA). Fetal bovine serum (FBS), RPMI-1640 
medium, and Dulbecco's phosphate-buffered saline (DPBS) were from Invitrogen (Carlsbad, CA, USA). Antibodies against $\mathrm{Rb}, \mathrm{Bax}, \mathrm{Bim}, \mathrm{Bcl}_{\mathrm{XL}}$, cyclin $\mathrm{E}$, cyclin $\mathrm{D} 1$, cyclindependent kinase (CDK) 2, CDK4, p21 $1^{\text {Wafl/Cip1 }}$, extracellular signal-regulated kinases (Erk) $1 / 2$ and $\beta$-actin were obtained from Cell Signaling Technology (Beverly, MA, USA). MRP1, BCRP and P-gp antibodies were purchased from Santa Cruz Biotechnology, Inc. (Santa Cruz, CA, USA) and anti-glyceraldehyde-3-phosphate dehydrogenase (GAPDH) antibody was obtained from Calbiochem (EMD Biosciences Inc., San Diego, CA, USA). Secondary antibodies were purchased from Bio-Rad (Hercules, CA, USA). Enhanced chemiluminescence solution (ECL) was kindly donated by Detroit R\&D, Inc. (Detroit, MI, USA). Propidium iodide and RNase A were from Abcam (Cambridge, UK).

Establishment of a butyrate-resistant cell line. The HCT116 human colon cancer cell line was obtained from the American Type Culture Collection (ATCC, Manassas, VA, USA) and cultured in RPMI-1640 medium supplemented with 10\% FBS and $1 \%$ penicillin-streptomycin at $37^{\circ} \mathrm{C}$ in a humidified $5 \% \mathrm{CO}_{2}$ incubator. To establish the butyrate-resistant HCT116 colon cancer cell line (HCT116/BR), HCT116 parental cells (HCT116/PT) were initially incubated with serum-containing media supplemented with $0.2 \mathrm{mM}$ sodium butyrate. Butyrate treatment induced the death of most cells while some cells survived, but they proliferated slower compared to HCT116/ PT cells. When cells were $80 \%$ confluent, the media was changed to a fresh media containing the same concentration of butyrate. By continuously growing cells in the presence of the same concentration of butyrate, no more detectable death of cells was observed and then the treating concentration of butyrate increased 2-fold. After culturing cells with the increasing concentration of sodium butyrate to a maximum of $1.6 \mathrm{mM}$ for 3 months, butyrate-resistant cells were established. Butyrate stock was prepared by dissolving sodium butyrate in DPBS. The treatment was carried out after overnight incubation to allow cell attachment.

Cell proliferation assay. Cell proliferation was determined in both cell lines according to a method described previously with slight modifications (9). HCT116/PT and HCT116/BR cells were seeded onto 96-well plates. Cells were treated with various concentrations of butyrate, paclitaxel, 5-fluorouracil (5-FU), doxorubicin and TSA. After 72-h incubation, medium was removed, $0.5 \%$ MTT stock was diluted 1:10 with medium and $100 \mu 1$ added to each well, followed by incubation at $37^{\circ} \mathrm{C}$ for a further $2 \mathrm{~h}$. Subsequently, dimethylsulfoxide (DMSO) was added to solubilize the purple formazan crystals and then absorbance at $540 \mathrm{~nm}$ was measured using an ELISA reader (Bio-Tek Instruments Inc., Winooski, VT, USA).

Flow cytometry. To assess cell cycle progression, HCT116/PT and HCT116/BR cells were plated onto 60-mm dishes. After overnight incubation, HCT116/PT cells were treated with $6.4 \mathrm{mM}$ butyrate for $24 \mathrm{~h}$. Cells were then trypsinized and centrifuged for $5 \mathrm{~min}$ at $500 \mathrm{xg}$ at $4{ }^{\circ} \mathrm{C}$, and fixed with $70 \%$ ethanol at $4^{\circ} \mathrm{C}$, followed by addition of $1 \mathrm{ml}$ of propidium iodide solution (final concentration, $50 \mu \mathrm{g} / \mathrm{ml}$ ), which contained $200 \mu \mathrm{g} / \mathrm{ml}$ RNase A, for 30 min in the dark (10). Flow cytometry was then performed using a FACSCalibur flow cytometer and identified using Cell Quest software (Becton-Dickinson, San Jose, CA, USA). Red fluorescence, measured at 585/542 nm, indicative of propidium iodide uptake by damaged cells, was measured by logarithmic amplification and electronic compensation for spectral overlap (11).

Protein extraction and immunoblotting. Cells were lysed with cell lysis buffer containing protease inhibitor cocktail, and protein concentration was determined using the bicinchoninic acid (BCA) assay. Protein samples (20-30 $\mu$ g per lane) were resolved by $7.5-12.5 \%$ sodium dodecyl sulfate polyacrylamide gel electrophoresis (SDS-PAGE) and transferred to a nitrocellulose membrane. For immunodetection, blots were incubated with 1:1,000 diluted primary antibodies in 5\% bovine serum albumin (BSA) Tris-buffered saline (TBS) containing $0.1 \%$ Tween-20 (TBS-T) at $4^{\circ} \mathrm{C}$ with gentle shaking overnight, followed by incubation with a secondary antibody conjugated to horseradish peroxidase at room temperature. The antigenantibody complexes were detected using ECL reagents with an ImageQuant LAS-4000 mini (GE Healthcare Life Sciences, Piscataway, NJ, USA). GAPDH or $\beta$-actin was used as the loading control (12).

Wound healing assay. Cells were seeded onto 6-well plates to create a confluent monolayer after overnight incubation. Mitomycin C was treated (final concentration, $25 \mu \mathrm{g} / \mathrm{ml}$ ) for $30 \mathrm{~min}$ and removed. A straight line was scratched in the middle of the cell monolayer. After washing with DPBS, cells were treated with chemotherapeutic drugs and incubated up to $24 \mathrm{~h}$. The widths of the scratched line were measured at appropriate time intervals and compared to that at $0 \mathrm{~h}$.

Gelatin zymography. Activity of matrix metalloproteinases (MMPs) was detected using gelatin zymography according to a method described previously with slight modifications (13). Briefly, cells were incubated in $60-\mathrm{mm}$ dishes and medium was replaced with serum-free medium to promote MMP secretion when cells reached $80 \%$ confluence. After 48-h incubation, medium was harvested and concentrated using a Speed-vac (Savant/E-C Instruments, Niantic, CT, USA). Electrophoresis was performed in 10\% SDS-PAGE gels containing $0.1 \%$ gelatin (Wako Pure Chemical Industries, Ltd., Osaka, Japan) without a reducing agent or boiling. After electrophoresis, the gel was soaked with renaturing buffer $(2.5 \%$ Triton X-100, v/v) for $1 \mathrm{~h}$, rinsed with distilled water three times, incubated with developing buffer $(50 \mathrm{mM}$ Trisbase, $150 \mathrm{mM} \mathrm{NaCl}, 1 \mu \mathrm{M} \mathrm{ZnCl}_{2}$ and $50 \mathrm{mM} \mathrm{CaCl}_{2}$ ) for $72 \mathrm{~h}$ at $37^{\circ} \mathrm{C}$, stained with $0.5 \%(\mathrm{w} / \mathrm{v})$ Coomassie Blue, and destained in destaining buffer (methanol:acetic acid:water, 10:5:85, v/v). Gel images were scanned using a Gel-Doc Imaging System (Bio-Rad) and band density was measured using the ImageJ software.

Statistical analysis. Data are presented as means \pm standard deviation (SD). Statistical analysis was performed using the GraphPad Prism 5 software. Data were compared using an unpaired two-tailed t-test or one-way analysis of variance (ANOVA), followed by Dunnett's post hoc test. P-values $<0.05$ were considered to indicate statistical significance (14). 

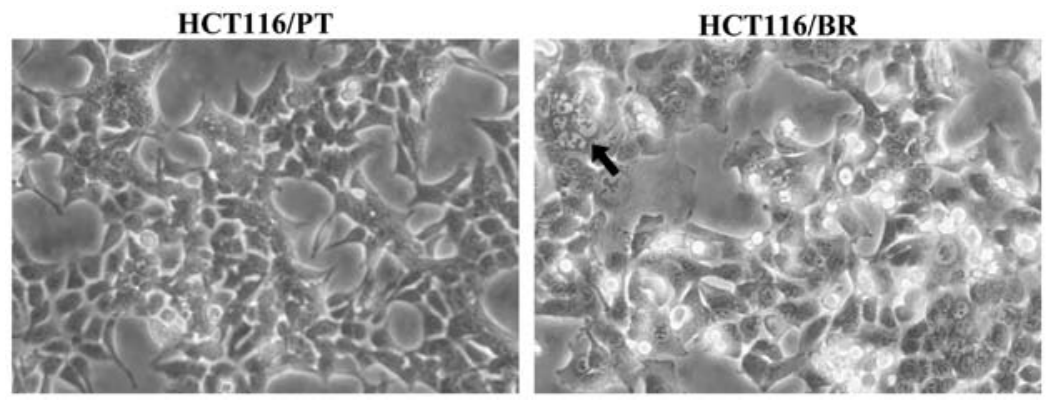

Figure 1. Micrographs of HCT116 parental (HCT116/PT) (left) and butyrate-resistant HCT116 (HCT116/BR) cells (right). Cell morphology was visualized by light microscopy. Magnification, x200. Arrow indicates vacuole.
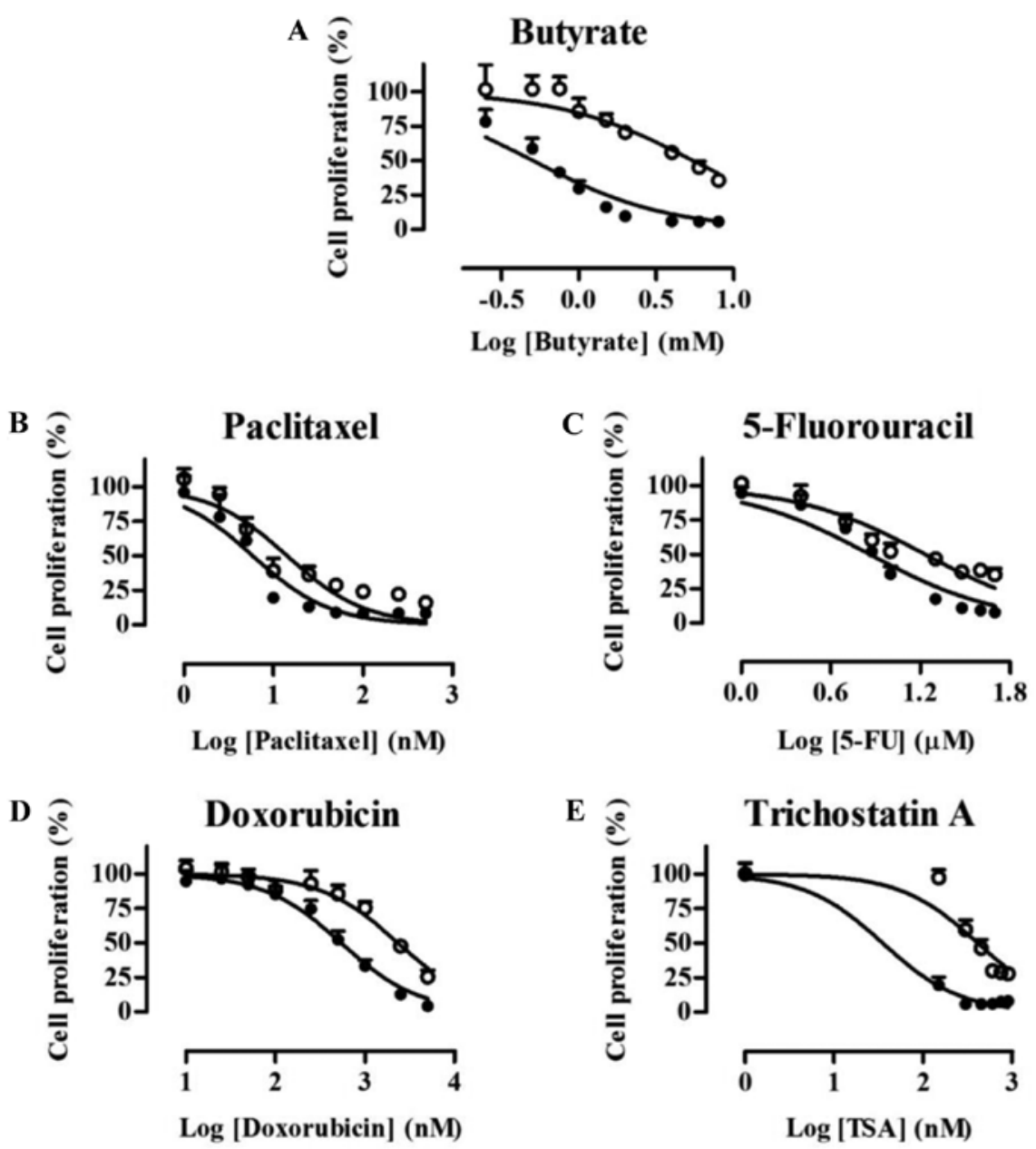

Figure 2. Cell proliferation determined by MTT assay. Effects of chemotherapeutic agents on HCT116 parental (HCT116/PT, $\bullet$ ) and butyrate-resistant HCT116 (HCT116/BR, o) cells. Dose-response curves for butyrate (A), paclitaxel (B), 5-fluorouracil (5-FU) (C), doxorubicin (D) and trichostatin A (TSA) (E) treatment for $72 \mathrm{~h}$. The data are means \pm standard deviation (SD) of triplicate determinations.

\section{Results}

Establishment of butyrate-resistant HCT116 cells.HCT116/BR cells were derived from the HCT116 human colon cancer cell line by chronic exposure to butyrate for 3 months. Cell morphology was altered slightly (Fig. 1). HCT116/PT cancer cells had cuboidal and sharp-pointed shapes, but HCT116/BR cells were more rounded and expanded. In addition, vacuolization was observed in HCT116/BR cells. The growth rate of the cells was lower than that of HCT116/PT cells.
Chemoresistance of HCT116/BR cells. Whether resistance to other chemotherapeutic agents accompanied resistance to butyrate was assessed by determining cell viability by MTT assay. HCT116/PT and HCT116/BR cells were treated with butyrate, paclitaxel, 5-FU, doxorubicin and TSA, and the dose-response curve and the concentrations that inhibited cell growth by $50 \%\left(\mathrm{IC}_{50}\right)$ values are shown in Fig. 2 and Table $\mathrm{I}$, respectively. The inhibition of cell proliferation was increased in a concentration-dependent manner in both cell lines, but HCT116/BR cells exhibited greater chemoresistance. The 


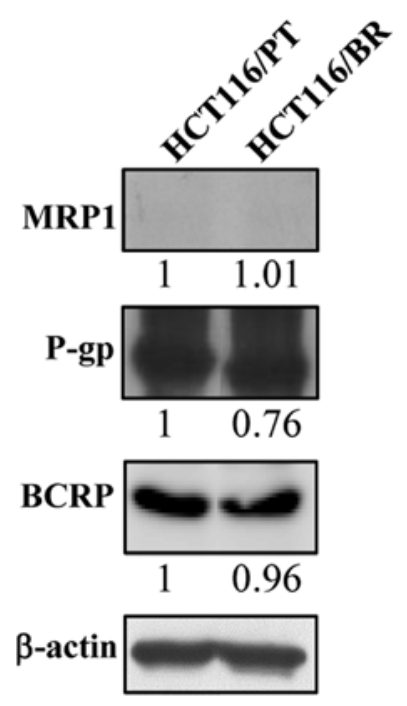

Figure 3. Expression of drug efflux pumps. Proteins related to the drug efflux pumps were analyzed by immunoblotting. Expression of the drug efflux pumps, P-glycoprotein (P-gp), breast cancer-resistant protein (BCRP) and the multidrug resistance associated protein 1 (MRP1) was compared in HCT116 parental (HCT116/PT) and butyrate-resistant HCT116 (HCT116/BR) cells $\beta$-actin was used as the loading control. Band density was estimated using the ImageJ software.
Table I. IC ${ }_{50}$ values of chemotherapeutic drugs in HCT116/PT and HCT116/BR cells.

\begin{tabular}{lcc}
\hline Chemotherapeutic drugs & HCT116/PT & HCT116/BR \\
\hline Butyrate (mM) & 0.508 & 5.50 \\
Paclitaxel (nM) & 5.89 & 14.3 \\
5-FU $(\mu \mathrm{M})$ & 7.28 & 17.1 \\
Doxorubicin (nM) & 541 & 2,330 \\
Trichostatin A(nM) & 36.3 & 412 \\
\hline
\end{tabular}

$\mathrm{IC}_{50}$, the concentrations inhibiting the cell growth by $50 \%$.

$\mathrm{IC}_{50}$ values of HCT116/BR cells for butyrate, paclitaxel, 5-FU, doxorubicin and TSA were 10.8, 2.42, 2.36, 4.31 and 11.3-fold higher, respectively, than those of HCT116/PT cells.

Drug efflux pump expression. Drug efflux pumps are related to drug resistance (8). One possible mechanism of chemoresistance to butyrate is regulation of the expression of drug efflux pumps. Therefore, we determined P-gp, BCRP and MRP1 protein levels by immunoblotting (Fig. 3). There was no

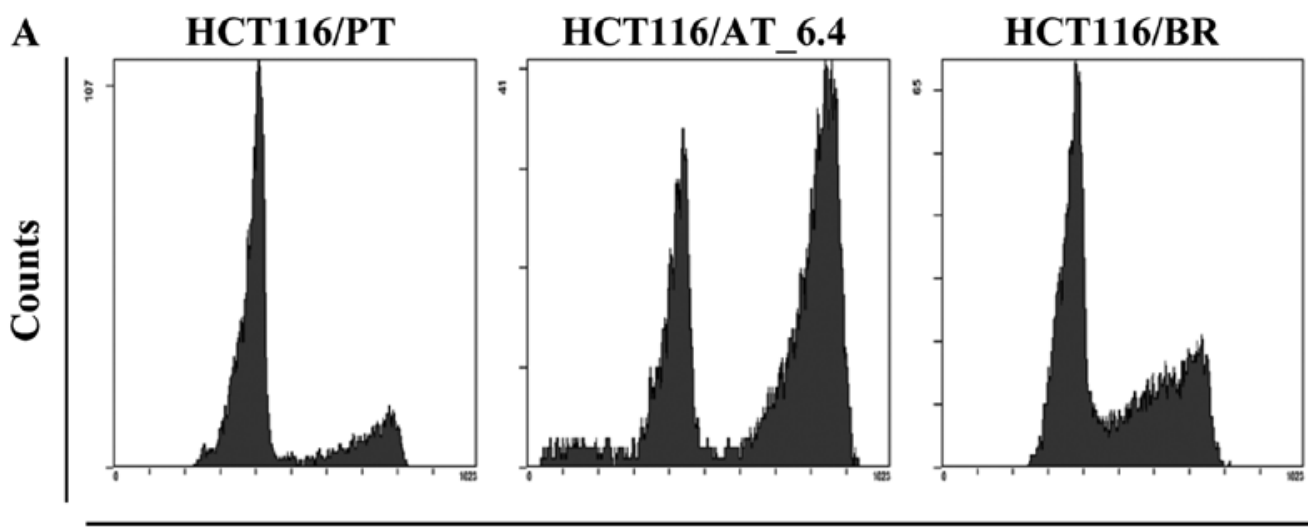

Fluorescence intensity

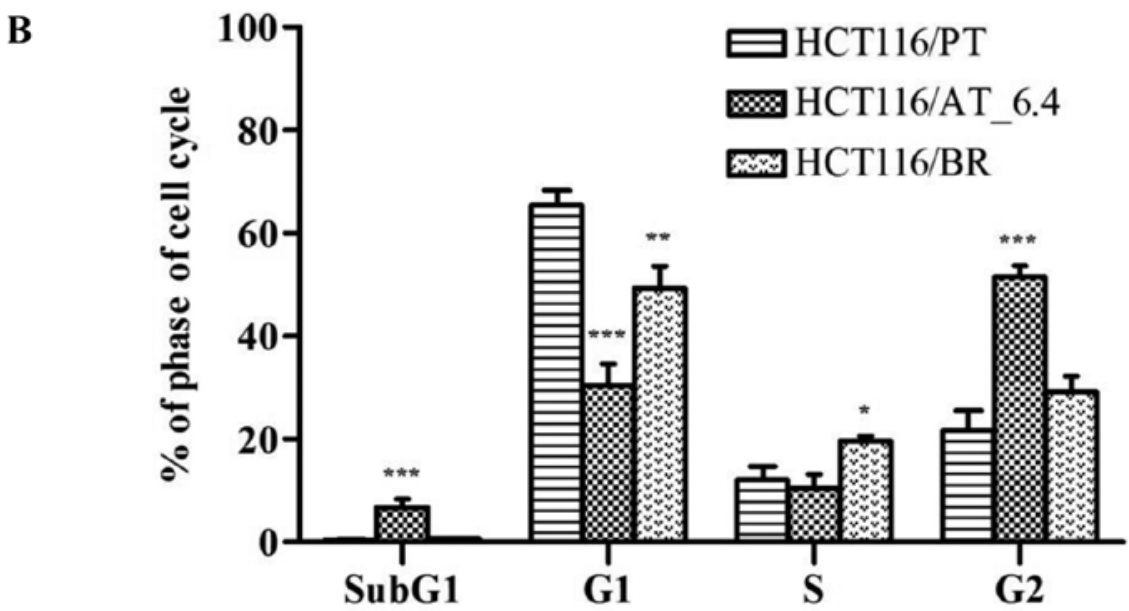

Figure 4. Relative DNA content according to cell cycle stage. Cell cycle progression was analyzed by flow cytometry with propidium iodide staining using $1 \times 10^{4}$ HCT116 parental (HCT116/PT) and HCT116 cells treated with 6.4 mM butyrate for 24 h (HCT116/AT_6.4) and butyrate-resistant HCT116 (HCT116/BR) cells. (A) Histogram was obtained by flow cytometry. (B) Bar graph showing the relative cell cycle phase distribution of each sample. The data are means \pm standard deviation (SD) of triplicate determinations. ${ }^{*} \mathrm{P}<0.05 ;{ }^{* *} \mathrm{P}<0.01 ;{ }^{* * *} \mathrm{P}<0.001$. 


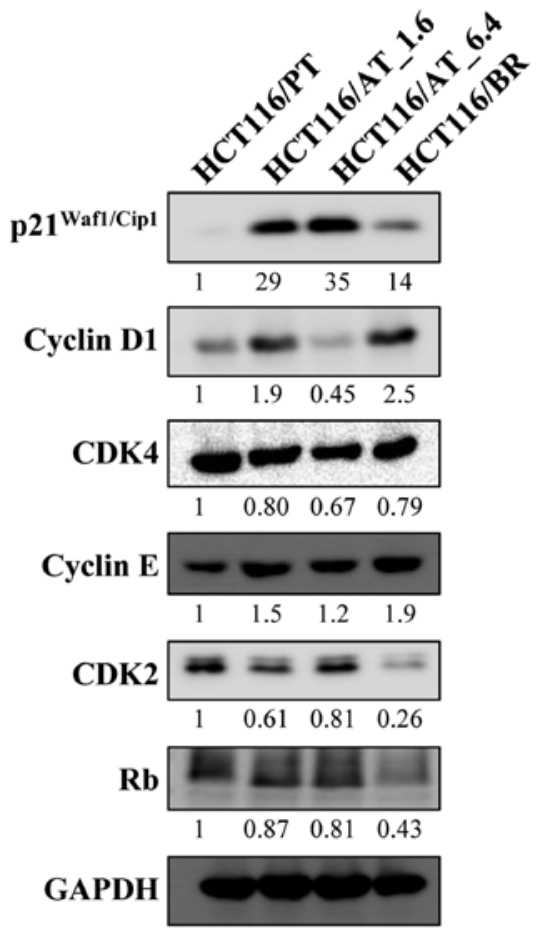

Figure 5. Expression of cell cycle-related proteins. Proteins related to the cell cycle were analyzed by immunoblotting. Protein expression was compared in HCT116 parental (HCT116/PT) and HCT116 cells treated with $6.4 \mathrm{mM}$ butyrate for 24 h (HCT116/AT_6.4) and butyrate-resistant HCT116 (HCT116/BR) cells. Glyceraldehyde 3-phosphate dehydrogenase (GAPDH) was used as the loading control. Band density was estimated using the ImageJ software.

significant difference in P-gp and BCRP expression between the cell lines, and MRP1 was not detected in HCT116/PT or HCT116/BR cells. Thus, drug efflux pumps did not affect the chemoresistance of HCT116/BR cells.

Induction of cell cycle arrest by butyrate. Butyrate induces cell cycle arrest and apoptosis in cancer cells (15), but our findings indicated no such effects in HCT116/BR cells. We performed flow cytometry to assess the effect of butyrate on cell cycle progression in HCT116/BR cells (Fig. 4). We also treated HCT116/PT cells with $6.4 \mathrm{mM}$ butyrate (4-fold of $1.6 \mathrm{mM}$ ) for $24 \mathrm{~h}$ (HCT116/AT_6.4) and compared the results with those of HCT116/PT and HCT116/BR cells. The proportion of subG ${ }_{1-}$ phase cells was increased by treatment with $6.4 \mathrm{mM}$ butyrate compared to untreated HCT116/PT cells (6.69 vs. $0.47 \%$ ) suggesting that apoptosis was triggered. The proportion of subG $_{1}$-phase $\mathrm{HCT} 116 / \mathrm{BR}$ cells was similar $(0.68 \%)$. The proportion of cells in $\mathrm{S}$ phase, which is a DNA-replication phase of the cell cycle, was elevated by $62.0 \%$ in HCT116/BR compared to HCT116/PT cells. In addition, the proportion of HCT116/AT_6.4 cells in $\mathrm{G}_{2}$ phase was >2-fold greater than that of HCT116/PT cells (51.4 vs. 21.6\%), but was restored to the control level in HCT116/BR cells (29.1\%).

Protein expression on cell cycle and apoptosis. We conducted an immunoblotting analysis to examine the expression of proteins involved in the cell cycle and apoptosis in HCT116/PT and HCT116/BR cells. We treated HCT116 cells

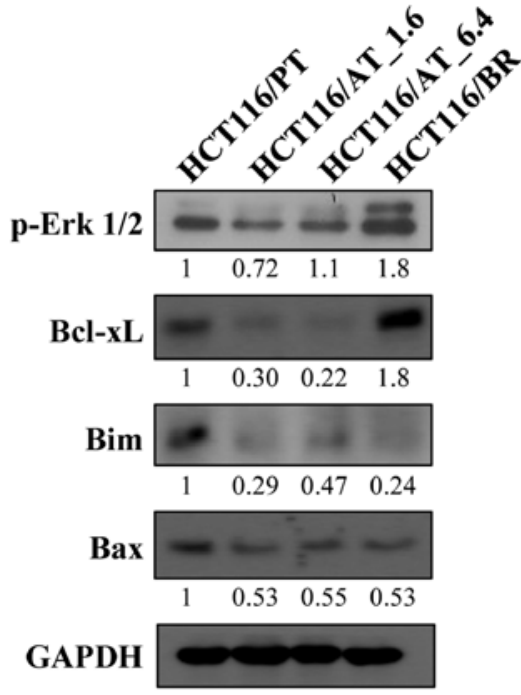

Figure 6. Expression of apoptosis-related proteins. Expression of phosphorylated extracellular signal-regulated kinase 1/2 (p-Erk1/2) and Bcl-2 family members was determined by immunoblotting. Protein expression was compared in HCT116 parental (HCT116/PT) and HCT116 cells treated with 6.4 mM butyrate for $24 \mathrm{~h}$ (HCT116/AT_6.4) and butyrate-resistant HCT116 (HCT116/BR) cells. Glyceraldehyde 3-phosphate dehydrogenase (GAPDH) was used as the loading control. Band density was estimated using the ImageJ software.

with 1.6 and $6.4 \mathrm{mM}$ butyrate for $24 \mathrm{~h}$ (HCT116/AT_1.6 and HCT116/AT_6.4, respectively) to determine the effects of acute butyrate treatment.

Expression of $\mathrm{p} 21^{\mathrm{Waf} 1 / \mathrm{Cip} 1}$ was greatly increased in HCT116/ AT_1.6 and HCT116/AT_6.4 cells, but the increase was less marked in HCT116/BR cells compared to HCT116/PT cells (Fig. 5). HCT116/BR cells showed greater cyclin D1 expression compared to HCT116/PT cells. CDK4 protein expression was minimally changed. $\mathrm{Rb}$ expression was decreased in HCT116/BR cells. Cyclin E expression in HCT116/BR cells was slightly increased by butyrate; however, CDK2 expression was greatly reduced.

The Bcl-2 family, which includes Bcl-xL, Bim and Bax, is involved in the regulation of apoptosis, and their expression is shown in Fig. 6. The expression of Bcl-xL, which is an important anti-apoptotic protein, was decreased in HCT116/ AT_1.6 and HCT116/AT_6.4 cells by the acute butyrate treatment, but was significantly increased in HCT116/BR cells. Bim and Bax are pro-apoptotic proteins, the expression of which was decreased in HCT116/AT_1.6, HCT116/AT_6.4 and HCT116/BR cells by both chronic and acute butyrate treatment. Moreover, activation of Erk reduces the expression of Bim, and phospho-Erk1/2 expression was increased in HCT116/BR cells.

Effect of butyrate on invasiveness. We performed gelatin zymography and a wound healing assay to evaluate cell invasiveness (Fig. 7) and migratory activity (Fig. 8). The wound healing rate was generally slower in HCT116/BR than HCT116/PT cells. MMP-9 plays a role in cancer cell metastasis, and there was no significant difference in its expression between HCT116/PT and HCT116/BR cells (Fig. 7). However, following short-term butyrate treatment, MMP-9 expression 

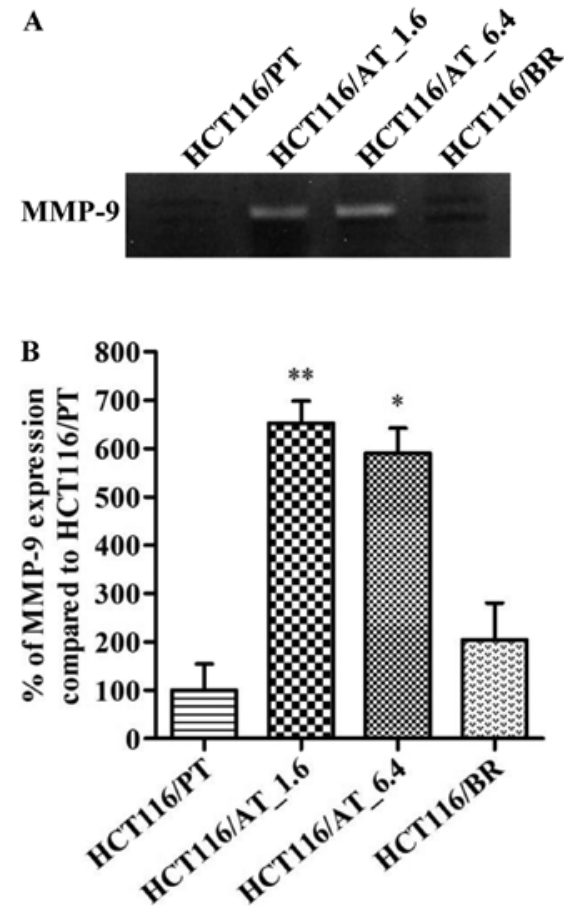

Figure 7. Gelatin zymography of matrix metalloproteinase-9 (MMP-9). MMP-9 expression was compared in HCT116 parental (HCT116/PT) and HCT116 cells treated with 1.6 (HCT116/AT_1.6) and $6.4 \mathrm{mM}$ butyrate (HCT116/AT_6.4) for $24 \mathrm{~h}$ and butyrate-resistant HCT116 (HCT116/BR) cells. (A) MMP-9 expression after 72-h development. (B) Quantification of band density using the ImageJ software. The data are means \pm standard deviation (SD) of triplicate determinations. ${ }^{*} \mathrm{P}<0.05 ;{ }^{* *} \mathrm{P}<0.01$. was significantly increased in both HCT116/AT_1.6 and HCT116/AT_6.4 cells. MMP-2 was not detected in HCT116/ PT and HCT116/BR cells. There was no significant difference in wound healing rate between HCT116/PT and HCT116/BR cells following treatment with butyrate, paclitaxel and TSA; however, doxorubicin significantly inhibited wound healing in both cell types.

\section{Discussion}

Butyrate inhibits proliferation and induces apoptosis of cancer cells, although it promotes the growth of normal cells. Recently, however, butyrate has been noted in colon cancer cells but the mechanism is unclear. Investigating the mechanism of resistance to butyrate in colon cancer cells is important for establishing new therapeutic strategies (16). In this study, we assessed the effect of resistance induced by butyrate in HCT116 human colon cancer cells.

We established butyrate-resistant HCT116 cells by chronic exposure to butyrate, which altered several of the cellular characteristics. First, chronic exposure of HCT116/BR cells to butyrate resulted in chemoresistance. Butyrate-resistant BCS-TC2.BR2 human colon adenocarcinoma cells are more tolerant to apoptosis induced by exogenous stimulation (7). Using a cell proliferation assay, we found that HCT116/BR cells had enhanced survivability compared to HCT116/PT cells following treatment with chemotherapeutic agents, such as paclitaxel, doxorubicin, TSA and 5-FU, as well as butyrate.
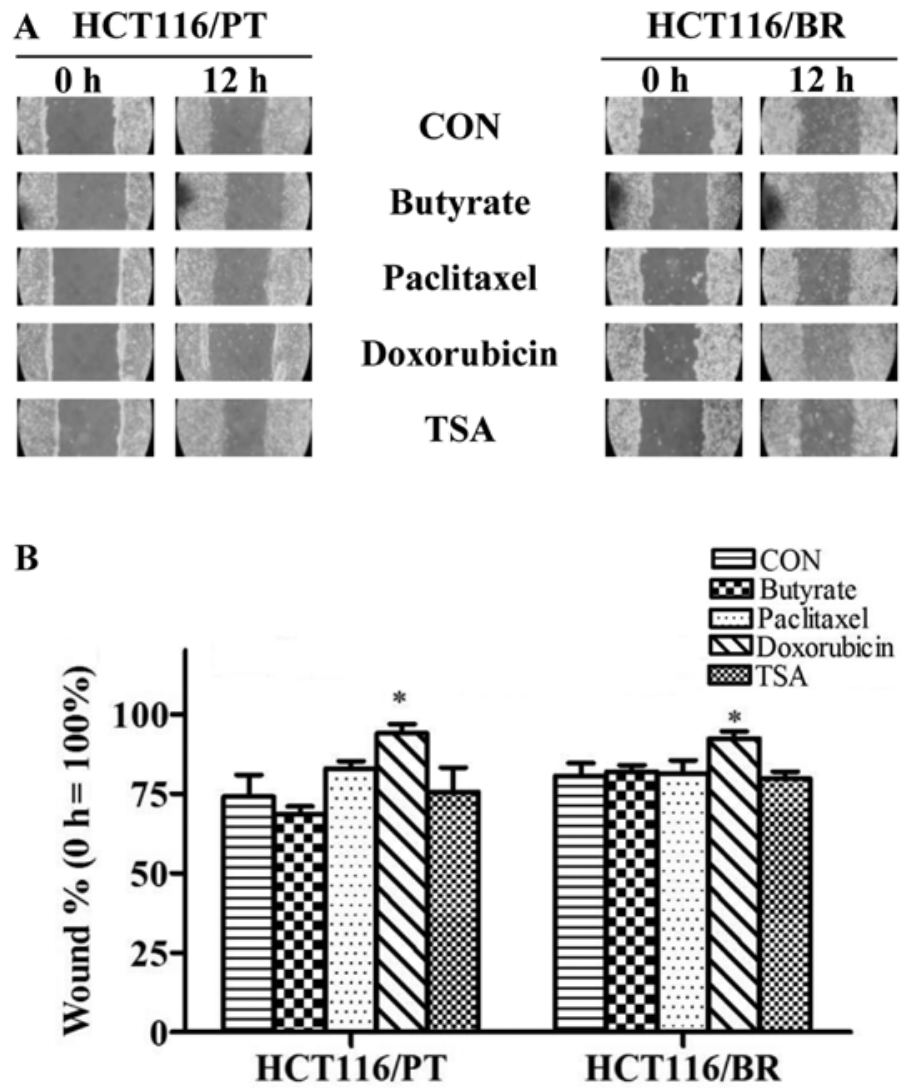

Figure 8. Effect of butyrate on wound healing. Wound healing assays of butyrate $(2 \mathrm{mM})$, paclitaxel $(100 \mathrm{nM})$, doxorubicin $(10 \mu \mathrm{M})$ and TSA $(300 \mathrm{nM})$ were performed in HCT116 parental (HCT116/PT) and butyrate-resistant HCT116 (HCT116/BR) cells. (A) Images taken at $12 \mathrm{~h}$ after drug treatments. (B) Wound percentages were calculated as the width at $12 \mathrm{~h}$ relative to that at $0 \mathrm{~h}$ for each treatment. The data are means \pm standard deviation (SD) of triplicate determinations. ${ }^{\mathrm{P}}<0.05$. 
This suggests that resistance to butyrate is accompanied by tolerance to chemotherapeutic agents. We hypothesized drug efflux pumps to be a possible resistance mechanism. However, there were no significant differences in the protein levels of drug efflux pumps, such as P-gp, BCRP and MRP1. Consequently, multidrug resistance in butyrate-resistant HCT116/BR colon cancer cells is not associated with resistance to chemotherapeutic agents.

We next examined the effect of butyrate on cell cycle progression. The proportion of HCT116/PT cells in $\mathrm{G}_{1}$ phase was higher, and that in $\mathrm{G}_{2}$ phase lower compared to HCT116/ AT_6.4 cells. Following short-term treatment with butyrate, the proportion in $\mathrm{G}_{2}$ phase was significantly increased in HCT116/AT_6.4 cells; however, HCT116/BR cells exhibited a pattern similar to the parental cells, suggesting that chronic exposure to butyrate acquired the resistance to butyrate and thus resulted in restoration of a normal cell cycle progression.

Protein expression data suggested that escape of cell cycle arrest induced by butyrate in HCT116/BR cells was dependent on $\mathrm{p} 21^{\mathrm{Waf} 1 / \mathrm{Cip} 1}$, cyclin D1 and Rb signaling. A previous study reported that HeLa human cervical cancer cells showed resistance to butyrate, which was associated with upregulation of cyclin D1 (17). Butyrate induced $\mathrm{G}_{1}$ arrest by disturbing $\mathrm{Rb}$ signaling was mediated by inhibition of cyclin D1 and p53-independent induction of p21 ${ }^{\text {Waf1/Cip1 }}$ (3). In contrast, CDK2 expression was significantly decreased in HCT116/BR cells. This was supported by the flow cytometry results, which indicated an increased proportion of HCT116/BR cells in S phase, likely because the cyclin E/CDK2 complex is required for progression from $G_{1}$ to $S$ phase (18). Furthermore, inhibition of apoptosis was confirmed in HCT116/BR cells by upregulation of Bcl-xL, and downregulation of Bim and Bax, as well as upregulation of phospho-Erk, which is a regulator of Bim $(4,16)$. This is a unique mechanism that activation of Erk1/2 induces the phosphorylation of Bim, leads Bim to degradation via the proteasome and results in the protection against cell death $(19,20)$.

We also examined MMP-9 expression to determine the effect of butyrate on invasiveness. MMP-9 expression was increased by acute butyrate treatment for $72 \mathrm{~h}$. In contrast, MMP-9 expression in HCT116/BR cells was negligible and comparable to that of HCT116/PT cells. The mechanism underlying the increased MMP-9 expression in HCT116/AT cells was unclear; however, MMP-9 expression was reported to be increased in the HCT15 colon cancer cell line by treatment with $1 \mathrm{mM}$ butyrate for 5 days (21). Although short-term butyrate treatment results in upregulation of MMP-9 expression, it was gradually decreased to the level of parental cells by continuous exposure to butyrate. This was confirmed by a wound-healing assay, in which no significant differences were observed between HCT116/PT and HCT116/BR cells. Therefore, chronic butyrate treatment did not have a marked effect on cell invasiveness and migration, despite the fact that acute butyrate treatment influenced MMP-9 expression.

Collectively, we assessed butyrate resistance and noted several similarities between HCT116/PT and HCT116/BR cells. Following acute butyrate treatment, the proportion of cells in $\mathrm{G}_{2}$ phase was highly increased, but recovered to the level of the control in HCT116/BR cells. However, there were several differences between HCT116/BR compared to
HCT116/PT cells. HCT116/BR cells exhibited considerable chemoresistance, which was not mediated by drug efflux pumps. Resistance to cell cycle arrest and apoptosis induced by butyrate in HCT116/BR cells was related to the regulation of Bcl-2 family proteins and cyclin D1 as well as $\mathrm{p} 21^{\text {Waf1/Cip1. }}$

\section{Acknowledgements}

This study was supported by Basic Science Research Program through the National Research Foundation of Korea (NRF) funded by the Ministry of Science, ICT and future Planning (NRF-2015R1A2A2A01007546).

\section{References}

1. Siegel RL, Miller KD and Jemal A: Cancer statistics, 2015. CA Cancer J Clin 65: 5-29, 2015.

2. Gaschott T and Stein J: Short-chain fatty acids and colon cancer cells: The vitamin D receptor - butyrate connection. Recent Results Cancer Res 164: 247-257, 2003.

3. Vaziri C, Stice L and Faller DV: Butyrate-induced G1 arrest results from p21-independent disruption of retinoblastoma protein-mediated signals. Cell Growth Differ 9: 465-474, 1998.

4. Ruemmele FM, Schwartz S, Seidman EG, Dionne S, Levy E and Lentze MJ: Butyrate induced Caco-2 cell apoptosis is mediated via the mitochondrial pathway. Gut 52: 94-100, 2003.

5. Guilloteau P, Martin L, Eeckhaut V, Ducatelle R, Zabielski R and Van Immerseel F: From the gut to the peripheral tissues: The multiple effects of butyrate. Nutr Res Rev 23: 366-384, 2010.

6. Donohoe DR, Collins LB, Wali A, Bigler R, Sun W and Bultman SJ: The Warburg effect dictates the mechanism of butyrate-mediated histone acetylation and cell proliferation. Mol Cell 48: 612-626, 2012.

7. López de Silanes I, Olmo N, Turnay J, González de Buitrago G, Pérez-Ramos P, Guzmán-Aránguez A, García-Díez M, Lecona E, Gorospe M and Lizarbe MA: Acquisition of resistance to butyrate enhances survival after stress and induces malignancy of human colon carcinoma cells. Cancer Res 64: 4593-4600, 2004.

8. Leslie EM, Deeley RG and Cole SP: Multidrug resistance proteins: Role of P-glycoprotein, MRP1, MRP2, and BCRP (ABCG2) in tissue defense. Toxicol Appl Pharmacol 204: 216-237, 2005.

9. Lepiarczyk M,KałużaZ,Bielawska A,Czarnomysy R, Gornowicz A and Bielawski K: Cytotoxic activity of octahydropyrazin[2,1-a:5,4a']diisoquinoline derivatives in human breast cancer cells. Arch Pharm Res 38: 628-641, 2015.

10. Han YS, Lee JH and Lee SH: Antitumor effects of fucoidan on human colon cancer cells via activation of Akt signaling. Biomol Ther (Seoul) 23: 225-232, 2015.

11. Lim SJ, Choi HG, Jeon CK and Kim SH: Increased chemoresistance to paclitaxel in the MCF10AT series of human breast epithelial cancer cells. Oncol Rep 33: 2023-2030, 2015.

12. Song HM, Park GH, Eo HJ, Lee JW, Kim MK, Lee JR, Lee MH, Koo JS and Jeong JB: Anti-proliferative effect of naringenin through p38-dependent downregulation of cyclin D1 in human colorectal cancer cells. Biomol Ther (Seoul) 23: 339-344, 2015.

13. Zhang R, Pan X, Huang Z, Weber GF and Zhang G: Osteopontin enhances the expression and activity of MMP-2 via the SDF-1/ CXCR4 axis in hepatocellular carcinoma cell lines. PLoS One 6: e23831, 2011

14. Cook KL, Shajahan AN, Wärri A, Jin L, Hilakivi-Clarke LA and Clarke R: Glucose-regulated protein 78 controls cross-talk between apoptosis and autophagy to determine antiestrogen responsiveness. Cancer Res 72: 3337-3349, 2012.

15. Gonçalves P and Martel F: Butyrate and colorectal cancer: The role of butyrate transport. Curr Drug Metab 14: 994-1008, 2013.

16. Barrasa JI, Santiago-Gómez A, Olmo N, Lizarbe MA and Turnay J: Resistance to butyrate impairs bile acid-induced apoptosis in human colon adenocarcinoma cells via up-regulation of Bcl-2 and inactivation of Bax. Biochim Biophys Acta 1823: 2201-2209, 2012

17. Derjuga A, Richard C, Crosato M, Wright PS, Chalifour L, Valdez J, Barraso A, Crissman HA, Nishioka W, Bradbury EM, et al: Expression of p21Waf1/Cip1 and cyclin D1 is increased in butyrate-resistant HeLa cells. J Biol Chem 276: 37815-37820, 2001. 
18. Zhang Y, Wang Z, Ahmed F, Banerjee S, Li Y and Sarkar FH: Down-regulation of Jagged-1 induces cell growth inhibition and S phase arrest in prostate cancer cells. Int J Cancer 119: 2071-2077, 2006.

19. Ley R, Balmanno K, Hadfield K, Weston C and Cook SJ: Activation of the ERK1/2 signaling pathway promotes phosphorylation and proteasome-dependent degradation of the BH3-only protein, Bim. J Biol Chem 278: 18811-18816, 2003.

20. Luciano F, Jacquel A, Colosetti P, Herrant M, Cagnol S, Pages G and Auberger P: Phosphorylation of Bim-EL by Erk1/2 on serine 69 promotes its degradation via the proteasome pathway and regulates its proapoptotic function. Oncogene 22: 6785-6793, 2003.
21. Serpa J, Caiado F, Carvalho T, Torre C, Goncalves LG, Casalou C, Lamosa P, Rodrigues M, Zhu Z, Lam EWF, et al: Butyrate-rich colonic microenvironment is a relevant selection factor for metabolically adapted tumor cells. J Biol Chem 285: 39211-39223, 2010. 